\title{
HD 209458: Physical Parameters of the Parent Star and the Transiting Planet
}

\author{
Ann Marie Cody \& Dimitar D. Sasselov ${ }^{1}$ \\ Dept. of Astronomy, Harvard University, 60 Garden St., Cambridge MA 02138
}

\begin{abstract}
The Sun-like star HD 209458 harbors a close-in giant planet that transits across the star's disk, and thus allows an unprecedented access to the basic parameters of the planet, given a certain knowledge of the basic parameters of the star, namely its mass and radius. We present theoretical stellar evolution model calculations for HD 209458 and discuss the uncertainties involved in deriving the stellar mass and radius. We derive the mass, $M=1.06 M_{\odot}$, radius, $R=1.18 R_{\odot}$, and age, $t=5.2 \mathrm{Gyr}$ of the star with uncertainties of $10 \%$ or more. The dominant sources of uncertainty remain to be the helium abundance estimate and the treatment of convection, even after an optimistic estimate for the effective temperature of the star. However, we find that in deriving the radius of the planet, $R_{p}$, the relevant stellar model input is the $\mathrm{M} / \mathrm{R}$ relation, which runs orthogonal to a degeneracy in the transit light curve solution and greatly improves the estimate of $R_{p}$. Theoretically the $\mathrm{M} / \mathrm{R}$ relation has a lower uncertainty than the $M$ and $R$ separately. We estimate the planet radius and mass to be $R_{p}=1.42_{-0.13}^{+0.10} R_{J}$ and $M_{p}=0.69 \pm 0.02 M_{J}$.
\end{abstract}

Subject headings: extrasolar planetary systems; stars - evolution; stars HD 209458

\section{Introduction}

The transit detection of HD 209458 b (Charbonneau et al. 2000; Henry et al. 2000) was a milestone in the study of extrasolar planets. Since the first close-in extrasolar giant planet (CEGP), 51 Peg b, was discovered in 1995 by Mayor \& Queloz (1995), the nature of these unusual type of objects was not truly understood. Eleven more close-in extrasolar giant

\footnotetext{
${ }^{1}$ Alfred P. Sloan Foundation Fellow
} 
planets with orbits $\leq 0.05 \mathrm{AU}$ are now known. However, thanks to the transit detection of HD 209458 b, we are certain now that they are indeed gas giant planets, much like our own Jupiter and Saturn. The transit of HD 209458 b fixes the orbital inclination (which removes the sin $i$ ambiguity in mass), it gives the planet radius, provides the average planet density, and confirms that the CEGPs are gas giants.

The crucial parameters for understanding the nature of the extrasolar planet HD 209458 b are its mass and radius, yet an accurate determination depends on our independent knowledge of the mass and radius of its parent star HD 209458 (Charbonneau et al. 2000; Mazeh et al. 2000). The latter is a solar-like star — a G0 subgiant (slightly more massive and evolved than our Sun), and our means to determine its basic physical parameters should be fairly good. However, the demands on precision are so high that current stellar evolution codes and model atmosphere analysis can hardly live up to the challenge. In this paper we make a detailed analysis of the stellar interior evolution models for HD 209458, relying on the Hipparcos parallax for its luminosity, L, and on the spectroscopic analysis of Mazeh et al. (2000) for its effective temperature, $T_{\text {eff }}$, and metallicity, $Z$. Our main goal is to quantify the uncertainties and systematic errors that are involved.

An alternative approach is possible: either with multi-color transit photometry (Jha et al. 2000; Deeg et a. 2001), or with very precise transit photometry (Brown et al. 2001). These studies assume that the stellar mass, $M$, is known and derive $R, R_{p}$, $i$, and $u$ (a one-parameter description of limb darkening). The procedure reduces the apparent uncertainty in $R_{p}$, but since $R$ and $i$ remain strongly correlated, we suggest an improved approach. Our approach relies on the astrophysical correlation between stellar $M$ and $R$, which breaks the degeneracy. In addition, it is a more robust result of stellar evolution theory than the mass estimate.

\section{The Model}

The star HD 209458 is extremely similar to our Sun from the point of interior modeling (Figure 1). Such close similarity allows us to use a theoretical model that is very well constrained for the Sun. For many parameters the changes to the HD 209458 interior model are small and we can use solar constraints to test their reliability, e.g., the size of the convection zone, etc. Our code is largely based on the Sienkiewicz, Paczynski, Ratcliff (1988) code as updated and distributed by R. Sienkiewicz, where our changes concern mainly the upper boundary and convection. It is a Henyey code that solves the equations of stellar structure in one dimension. We use the OPAL equation of state (Rogers et 
al. 1996) and the latest Livermore opacity tables (OPAL96, Iglesias \& Rogers, 1996) for the Grevesse \& Noels (1993) heavy element mixture. The tables are augmented by the Alexander \& Ferguson (1994) data on molecular and grain opacities. The nuclear reaction rates are calculated according to Bahcall \& Pinsonneault (1995). The diffusion of hydrogen and oxygen is treated after Thoul, Bahcall, \& Loeb (1994). The upper boundary is an atmosphere with a temperature distribution from model atmosphere integration and Kurucz (1992) opacities; for models very different from the present Sun, the Eddington $T-\tau$

relation is used. Convection is treated with the standard mixing-length prescription and the Schwarzschild stability criterion. Slow rigid-body rotation is allowed. The evolution of the abundances of $\mathrm{H},{ }^{3} \mathrm{He},{ }^{4} \mathrm{He},{ }^{14} \mathrm{~N},{ }^{16} \mathrm{O}$, and ${ }^{17} \mathrm{O}$ is followed. Magnetic fields are not considered.

The ZAMS models are computed with initial guessed values of the boundary parameters taken from the standard solar model for $M_{*}=1.0 M_{\odot}$ and scaled for masses in the Sun's vicinity. The subsequent iteration is similar to that used by Ford, Rasio, \& Sills (1999).

As a check we compared runs with the Yale Rotating Evolution Code (YREC) for as close as possible initial parameters and overall conditions prepared by D. Guenther (2000, private communication). Despite many differences in assumptions and numerics, the codes compare very well and we use the runs to study possible systematics in our theoretical HD 209458 model (see §3.3).

\section{Stellar Parameters}

\subsection{Observational Data}

While the evolution code is capable of outputting very precise values for the stellar properties in question, the accuracy of our results was contingent upon the quality of observational data. An examination of the literature revealed very few studies that have provided physical properties for HD 209458. Fortunately, however, our work followed on the heels of an extensive spectral analysis performed by Mazeh et al. (2000). They derive an effective temperature of $6000 \pm 50 \mathrm{~K}$ and a metallicity $([\mathrm{Fe} / \mathrm{H}])$ of $0.00 \pm 0.02$. We adopted these values but explored a wider range of heavy element abundances than the $[\mathrm{Fe} / \mathrm{H}]$ measurement error quoted above.

Another crucial parameter under consideration was the stellar luminosity. We have put to use recent data provided by the Hipparcos Space Astrometry Mission (ESA 1997, SP-1200), which measured an apparent V magnitude of 7.65 and a parallax of $21.14 \pm 1.00$ mas for HD 209458. Assuming negligible interstellar extinction, this implies an absolute magnitude of $4.28 \pm 0.10$ in $\mathrm{V}$. To transfer from magnitude to luminosity, we applied a 
bolometric correction (BC), relying on the $T_{\text {eff }} / \mathrm{BC}$ scales derived by Flower (1996). The star's derived temperature of $6000 \mathrm{~K}$ requires a correction factor of $-0.45 \pm 0.007$. These values indicate a bolometric magnitude of 4.23 , and hence a luminosity $1.61 \pm 0.15 L_{\odot}$ (or $0.208 \pm 0.040$ in logarithmic units).

\subsection{Theoretical Evolution Results}

With all of the requisite physical parameters in hand, we began the stellar modeling process. To guide interpretation of the program output, we constructed a portion of the H-R diagram with a central temperature/luminosity error box given by our adopted uncertainties (see figure 1). The limits of this box, 3.7745 to 3.7818 for temperature (log units), and 0.168 to 0.248 for luminosity (log units), indicate the narrow range of "target" values imposed by observation. In the search for plausible evolutionary tracks, we separated the code routine into runs of specific metallicities, including $Z=0.013,0.015,0.016$, $0.020,0.025$, and 0.031 . These abundancest were chosen based on the adopted metallicity range $[\mathrm{Fe} / \mathrm{H}]=0.00 \pm 0.1$. We elected to use the precise solar values $X_{\odot}=0.7059$ and $Z_{\odot}=0.0200$ (Guenther \& Demarque 1997), and we assumed the stellar initial hydrogen content $X=0.7$ for HD 209458. The parameters $Z=0.016$ and $Z=0.025$ were taken to be the lower and upper bounds of allowable metallicities, but a few models with the values 0.013 and 0.031 were used to test more extreme evolutionary behavior.

After computing a grid of models based on the chosen metal abundances, we determined the most favorable evolutionary tracks by retaining only those that met the strict temperature and luminosity requirements. The results of our modeling can be seen in figure 1; a number of theoretical tracks pass through the error box. In proceeding to run the evolutionary code on a wide variety of stellar parameters, we succeeded in associating with each metallicity a range of masses that yielded acceptable temperatures and luminosities. We have plotted tracks for the metallicities 0.016, 0.020, and 0.025 in figure 1. Masses shown for $Z=0.016$ are (in order of decreasing temperature) 1.009, 0.990, and $0.978 M_{\odot}$. The metallicity of 0.02 , constituting our best estimate for HD 209458 as well as the canonical solar value, was implemented for models of mass 1.15, 1.10, 1.09, 1.06, $1.05 M_{\odot}$ (the track for 1.10 is omitted from figure 1). It was also used in constructing a zero-age main sequence (ZAMS) track that can be seen cutting across the left portion of figure 1. The upper range of metallicities, 0.025, was run through the code with masses of

\footnotetext{
${ }^{2}$ Where $\log Z=\log \frac{X}{X_{\odot}}+\log Z_{\odot}+[F e / H]$, and $X_{\odot}, Z_{\odot}$ are the solar abundances of hydrogen and metals (by mass) for $X+Y+Z=1$.
} 
$1.175,1.13,1.12$, and $1.10 M_{\odot}(1.12$ is not plotted in figure 1$)$. A more complete set of data from the evolutionary tracks is available in table 1 . As evident from the H-R diagram, all of the metallicities we have selected have some range of masses that produces models reaching the desired temperature and luminosity (i.e. passing through the center of the error box). While not obvious from the figure, HD 209458 must have a metallicity as high as $Z=0.05$ (assuming solar hydrogen abundance) before its evolutionary models no longer intersect the error box. This situation allows for great freedom in choosing combinations of masses and abundances that match observational data. Therefore, narrowing down the parameters further required restrictions on mass and age.

The age of HD 209458 is not known; we could only put rough constraints on it from observed levels of stellar activity. For HD 209458 such constraints happen to make little difference, being somewhere between 4 and 7 Gyr of age (Mazeh et al. 2000). However, it is instructive to see how derived ages correlate with the possible range of stellar metallicity, and eliminate some models that are extraordinarily old or young. For a particular $Z$-value, we located three evolutionary tracks of differing mass: one passing directly through the target temperature of $6000 \mathrm{~K}$ and luminosity $1.61 L_{\odot}$, one that simultaneously reached the highest allowable temperature and lowest luminosity (thus giving the lowest age), and one that reached the highest luminosity and lowest temperature (giving the greatest age). We then noted the ages for which each model touched the limits of the error box. In the interest of making visual comparisons, we have plotted all metallicities versus these sets of age extremes in figure 2.

The results of our modeling reveal that not all of the theoretical evolutionary tracks for HD 209458 that pass through the temperature/luminosity error box evolve to a desirable age. On the scales that we are interested in, age range and $Z$ follow an approximately linear relationship. In fact, no models with metallicity greater than $0.030([\mathrm{Fe} / \mathrm{H}]=+0.18)$ or less than $0.012([\mathrm{Fe} / \mathrm{H}]=-0.22)$ can be considered viable. Therefore, we can be confident that HD 209458's metallicity is within these two values. Given the star's predicted solar metal abundance, this is hardly surprising; the theoretical models achieving optimal temperatures and luminosities are within a credible zone of abundances.

Both the evolutionary tracks and the analysis of stellar age range lend support to the original temperature and luminosity data for HD 209458: 6000K ( $\log =3.778)$ and $1.61 L / L_{\odot}(\log =0.208)$. These are the values at the center of our error box, and a model with $Z=0.02$ reaches them when computed with a mass of $1.06 M_{\odot}$. It should also be noted that models of mass 1.09 and $1.04 M_{\odot}$ just barely reach the range of reasonable temperatures and luminosities. All three models remain comfortably within the 4-7 Gyr range. The parameters $\mathrm{M}=1.06_{-0.02}^{+0.03} M_{\odot}, \log T_{\text {eff }}=3.778 \pm 0.004$, and 
$\log L / L_{\odot}=0.208 \pm 0.040$ then describe our best stellar model for HD 209458 with solar metallicity. If we allow for our assumed range of metallicities, $Z=0.02 \pm 0.005$ $([F e / H]=0.0 \pm 0.1)$, then the mass uncertainty increases to ${ }_{-0.09}^{+0.11}$. This is identical to the mass uncertainty $( \pm 0.10)$ estimated by Mazeh et al. (2000). Propagating all of our errors, we find a radius of $1.18_{-0.08}^{+0.07} R_{\odot}$. The age associated with this combination is 5.2 Gyr. Our next step is to take account of other systematic uncertainties.

\subsection{Uncertainties}

There are three large sources of uncertainty. They have to do with the stellar helium abundance (the surface amount of helium cannot be observed in cool stars), the diffusion of helium and other heavy elements, and the treatment of convection.

Helium. The initial abundance of helium, $Y$, is an important yet unobservable parameter that influences the stellar evolution model. Thus, we examined the effect of shifting the degree of our models' helium enhancements. Figure 3 illustrates the change induced in temperature when $Y$ is varied by 0.02 (from the original figure of 0.28 ), and metallicity is held at a constant $Z=0.02$. For a helium abundance of 0.26 , overall model temperature decreases, and the mass must be raised by $0.04 M_{\odot}$ in order for the evolutionary track to once again pass through the center of the temperature/luminosity error box. For a higher helium abundance of 0.30 , the opposite is true; temperature increases, and the model mass must be decreased by $0.04 M_{\odot}$ to achieve the optimal temperature and luminosity. In addition, an altered $Y$-value will cause a slight shift in the stellar age. Our models indicate that an increased helium abundance corresponds to an increase in age for models of similar temperature and luminosity. The best-fit model with $T_{\text {eff }}=6000 \mathrm{~K}$ and $\mathrm{L}=1.61 L_{\odot}$ increases to 5.7 Gyr for a helium abundance of 0.30 , and decreases to 4.7 Gyr for an abundance of 0.26. In summary, $Y$ contributes about $4 \%$ as a systematic uncertainty to the derived stellar mass for HD 209458.

Convection. In using a local prescription to compute the stellar convective envelope - the mixing length theory, we have to specify the free mixing length parameter, $\alpha$. Since $\alpha$ is a number unavailable observationally, we are left to surmise that HD 209458 is similar to the sun in its envelope convection properties. The comparable temperatures and metallicities of the two stars makes it likely that they share an $\alpha$ of 1.69 (which is the standard solar value (Guenther \& Demarque 1997). Nevertheless, it is instructive to investigate several evolutionary models with several different $\alpha$-values, following the findings of multi-dimensional radiation hydrodynamics models of stellar convection (e.g., Ludwig, Freytag, \& Steffen 1999). The evolutionary tracks plotted in figure 4 correspond 
to mixing length parameters of 1.40,1.55, 1.69 (solar), 1.85, and 2.0, and they reveal that variations in $\alpha$ do indeed have a noticeable effect. Increasing the value by 0.15 leads to an overall temperature increase of about $75 \mathrm{~K}$. The mass must be augmented by $0.03 M_{\odot}$ to force the model back through the center of the $T_{\text {eff }} / \mathrm{L}$ error box. For smaller $\alpha$ 's, the reverse trend occurs. Even more significant, however, are the shifts in age. For a set of models passing through the center of the box, decreasing the mixing length parameter to 1.40 pulls HD 209458's age down to 1.8 Gyr, and increasing $\alpha$ to 2.00 inflates it to 8.2 Gyr. Both numbers are clearly extreme values. The more moderate $\alpha=1.55$ and $\alpha=1.85$, on the other hand, produce modest jumps in age of about 1.5 Gyr. Although it is unlikely that extreme mixing length parameter values are the case, they are certainly incompatible with our established temperature and luminosity, unless $[\mathrm{Fe} / \mathrm{H}]$ is far different from solar. Therefore the unknown $\alpha$ contributes at the $3 \%$ level to the uncertainty of the stellar mass (and much more to the stellar age).

Diffusion. Another systematic uncertainty we can quantify is the magnitude of heavy element diffusion. Not accounting for the diffusion of helium produces the offset $(\sim 0.2 \%)$ shown in figure 1 between the known temperature and luminosity of the Sun and its theoretical evolution track. We ran the code for the standard solar values of $X=0.7059$, $Z=0.02$ (Guenther \& Demarque 1997), and $\log T_{\text {eff }}=3.7612$. A run with YREC reproduced precisely the effect as well. In summary, the systematic uncertainty due to diffusion is much less substantial (below 1\%), than the two discussed above.

To combine all uncertainties involved, we should note the following. The region of the H-R diagram inhabited by HD 209458 is populated by virtually vertical evolution tracks for our range of $Z$ and $Y$ (Figure 1). Therefore the stellar mass determination is subjected to the largest uncertainty, which could only be remedied by a better $T_{\text {eff }}$ or age derivation. Unfortunately, a better parallax would not help. A more accurate $T_{\text {eff }}$ cannot be derived spectroscopically at this time; even the $50 \mathrm{~K}$ uncertainty used here is quite optimistic. The systematic uncertainties due to $Y$ and $\alpha$ also affect primarily the stellar mass determination. Therefore we have finally: $M=1.06 \pm 0.10$ (obs) \pm 0.07 (sys) $M_{\odot}$ for HD 209458's mass. On the other hand, the stellar radius remains: $R=1.18 \pm 0.10 R_{\odot}$, and could be improved by a better distance to HD 209458, e.g., a FAME parallax. Clearly the linear correlation between $M$ and $R$ (i.e., the ratio $\mathrm{M} / \mathrm{R}$ ) in that small region of parameter space is the most accurate outcome of the theoretical models, because the model systematics are minimized. 


\subsection{Planetary parameters}

To derive the planetary radius $\left(R_{p}\right)$ and orbital inclination $(i)$ from the stellar radius and mass, we employed the light-curve data of Brown et al. (2001). Following the method discussed in Sackett (1999) and used by Charbonneau et al. (2000) and Mazeh et al. (2000), we constructed the appropriate limb-darkened transit models for HD 209458. For this particular analysis, we adopted the same limb darkening parameter, $c_{\lambda}$, as Mazeh et al. (2000): 0.56. In addition, we used an orbital period of 3.524739, obtained by Robichon \& Arenou (2000) through Hipparcos data. With these values, we produced a series of transit light curves for selected planetary radii and orbital inclinations and calculated the $\chi^{2}$ statistic for each, based on the Brown et al. (2001) photometry. For $R=1.18$ and $M=1.06$, we derive a planetary radius of $R_{p}=1.42_{-0.13}^{+0.10} R_{J}$, very close to the results of Mazeh et al. (2000) and Brown et al. (2001). The inclination angle, $i$, is $86.1_{-0.5}^{+1.1}$.

We can decrease the uncertainties in $R_{p}$ and $i$ by a factor of 2 for HD 209458 by using the theoretical $\mathrm{M} / \mathrm{R}$ ratio for the star. Two reasons are responsible. First, as we noted above, the relation minimizes two sources of systematic errors on the stellar mass. Second, and more importantly, the theoretical $\mathrm{M} / \mathrm{R}$ relation in this small region of the H-R diagram breaks a degeneracy in $R, i$, and $R_{p} / R$. The degeneracy is well-known in light curve solutions of detached eclipsing binary stars with a transit eclipse of a limb-darkened

primary (e.g., Popper 1984). The times of second and third contact are poorly determined, unless the limb darkening is precisely known (or zero). Often the mass of the primary star, $M$, is used to derive the orbital velocity of the secondary (unknown for our planet) in the calculation of the latitude of the transit. The transit latitude relates $M, R$, and $i$, and the best fit for $R_{p} / R$ is aligned along a positively correlated $M \& R$. This positive $\mathrm{M} / \mathrm{R}$ correlation can be seen in the HD 209458 transit curve solutions of HST photometry (Brown et al. 2001; their Fig. 5) and multi-color photometry (Deeg et al. 2001; their Fig. $5)$. The latter is $R=0.34 M+0.825( \pm 0.06)$. However, in the vicinity of HD 209458, the theoretical relation we compute has a negative slope, thus nicely constraining the transit curve solution, as shown in Figure 5. In the neighbourhood of the best value, we have:

$$
\frac{M}{M_{\odot}}-1.06=-0.96\left(\frac{R}{R_{\odot}}-1.18\right)-2.05(Y-0.28)+15.39(Z-0.02) .
$$

The correlation between stellar mass and radius for normal single stars near the main sequence (masses 1.0 to $1.1 M_{\odot}$ ) at constant luminosity would emerge from the definition of effective temperature. A hot main sequence star would be smaller than a cool main sequence star of the same $L, Y$, and $Z$ as shown in Figure 1. Of course, their ages will differ. Note that the observational determination of $R_{p}$, from the analysis of the transit light curve, is done by holding $L, Y$, and $Z$ fixed. With virtually vertical evolution tracks, 
the stellar mass, $M$, used in the solution, is practically independent of the stellar $L$ in the error box of HD 209458. Incidentally, $Z$ is mostly correlated to the $T_{\text {eff }}$ in its derivation, as well.

\section{Discussion \& Conclusion}

By exploring a wide variety of physical parameters characterizing HD 209458, we have been able to confirm the general validity of previous metallicity measurements as well as stellar mass and radius calculations, noting the consistencies in age within the regime of $T_{\text {eff }}=6000 \mathrm{~K}$ and $L / L_{\odot}=1.61$. Although the metallicity we have adopted may require adjustments, the models are enough to rule out the possibility that it is far different from solar. With a full set of evolutionary tracks in hand, we can hold the results of $M=1.06 \pm 0.13 M_{\odot}$ and $R=1.18 \pm 0.10 R_{\odot}$ to a high level of confidence. For comparison, the existing theoretical mass and radius determinations for HD 209458 make use of precalculated evolution model isochrones. Mazeh et al. (2000) used four different sets of models - by Schaller et al. (1992), Bertelli et al. (1994), Claret (1995), and by Yi, Demarque, \& Oemler (1997). And in a survey of several hundred thousand stars Allende Prieto \& Lambert (1999) derived a mass and a radius for HD 209458 using isochrones from Bertelli et al. (1994). Our results differ only in the acknowledgment of two sources of systematic error (unknown $Y$ and $\alpha$ ) which increase the mass and radius uncertainty for HD 209458.

The availability of new, precise transit photometry (Brown et al. 2001) only reinforces further the need for better stellar structure models. Our analysis shows that the relevant model input for solving transit light curves is the relation between stellar mass and radius in $§ 3.4$. For future transit systems, such mass-radius relations should be derived uniquely from the corresponding evolution models for the parent star. From our derived inclination angle $i$ of $86.1^{\circ}$, we obtain a planetary mass (dependent on our estimated stellar mass, $1.06 M_{\odot}$ ) of $0.685 \pm 0.02 M_{J}$. With the derived radius, $1.42_{-0.13}^{+0.10} R_{J}$, the planet density would be only $0.30 \mathrm{~g} \mathrm{~cm}^{-3}$.

As noted by Burrows et al. (2000), the fact that HD 209458 b has a radius-to-mass ratio sufficiently higher than that of Jupiter confirms the slowing of the radial shrinking process due to stellar insolation. They also point out that such a large radius requires the planet to have migrated inward at a fairly early age if it were formed at a distance beyond 0.5AU. A better handle on the planetary radius should allow distinguishing between theoretical evolutionary models for the planet itself, given different overall albedos. With the discovery of more transiting planets in the near future and with the Kepler and COROT missions, 
our ability to derive good planetary parameters (radii, masses, \& densities) will depend even more critically on the theoretical stellar models. The stellar $\mathrm{M} / \mathrm{R}$ relation is robust along most of the main sequence, and should provide the best constraint for transit light curve solutions and $R_{p}$ determination.

We are grateful to D. Guenther for providing special runs with YREC for code comparisons. We thank D. Charbonneau for making available to us the HST transit light curve before publication. We are grateful to D. Latham, R. Noyes and D. Charbonneau for reading the manuscript and many helpful discussions. Thanks to the referee, P. Eggleton, for the good suggestions. AMC wishes to thank the Harvard College Research Program for their continuing support of her work; DDS acknowledges support from the Alfred P. Sloan Foundation.

\section{REFERENCES}

Alexander D.R., Ferguson J.W., 1994, ApJ 437, 879

Allende Prieto C., \& Lambert, D.L. 1999, A\&A, 352, 555

Brown, T.M. et al. 2001, ApJ, 552, 699

Bahcall J.N., Pinsonneault M.H., 1995, Rev. Mod. Phys. 67, 781

Bertelli, G. et al. 1994, A\&AS, 106, 275

Burrows, A. et al. 2000, ApJ, 534, L97

Charbonneau, D. et al. 2000, ApJ, 529, L45

Claret, A. 1995, A\&AS, 109, 441

Deeg, H.J., Garrido, R., Claret, A. 2001, New Astronomy, 6, 51

Flower, P.J. 1996, ApJ, 469, 355

Ford, E.B., Rasio, F.A., \& Sills, A. 1999, ApJ, 514, 411

Grevesse N., Noels A., 1993, Phys. Scripta T47, 133

Guenther, D., \& Demarque, P. 1997, ApJ, 484, 937

Henry, G., et al. 2000, ApJ, 529, L41

Iglesias C.A., Rogers F.J., 1996, ApJ 464, 943

Jha, S. et al. 2000, ApJ, 540, L45 
Kurucz, R. 1992, in Stellar Population of Galaxies, IAU Symp. 149, eds. Barbuy \& Renzini (Dordrecht: Kluwer), 225

Ludwig, H.-G., Freytag, B., Steffen, M. 1999, A\&A, 346, 111

Mayor, M., \& Queloz, D. 1995, Nature, 378, 355

Mazeh, T. et al. 2000, ApJ, 532, L55

Popper, D.M. 1984, AJ, 89, 132

Robichon, N., \& Arenou, F. 2000, A\&A, 355, 295

Rogers F.J., Swenson F.J., Iglesias C.A., 1996, ApJ 456, 902

Sackett, P.D. 1999, in Planets Outside the Solar System: Theory and Observations, ed. J.-M. Mariotti \& D.M. Alloin (NATO/ASI Ser. C; Dordrecht: Kluwer), 189

Schaller, G., et al. 1992, A\&AS, 96, 269

Sienkiewicz, R., Paczynski, B., Ratcliff, S. 1988, ApJ, 326, 392

Thoul A.A., Bahcall J.N., Loeb A., 1994, ApJ 421, 828

Yi, S., Demarque, P., Oemler, A. 1997, ApJ, 486, 201 
Table 1. Evolutionary Model Data

\begin{tabular}{|c|c|c|c|}
\hline $\log T_{\text {eff }}(\mathrm{K})$ & $\log L / L_{\odot}$ & $R / R_{\odot}$ & $\log$ Age (yrs.) \\
\hline \multicolumn{4}{|c|}{$M=1.30 M_{\odot},[\mathrm{Fe} / \mathrm{H}]=0.25, X=0.70, Y=0.28, Z=0.035, \alpha=1.69$} \\
\hline 3.7830 & 0.2288 & 1.1797 & 0.0000 \\
\hline 3.7831 & 0.2443 & 1.2004 & 8.2425 \\
\hline 3.7837 & 0.3398 & 1.3362 & 9.3603 \\
\hline \multicolumn{4}{|c|}{$M=1.25 M_{\odot},[\mathrm{Fe} / \mathrm{H}]=0.25, X=0.70, Y=0.28, Z=0.035, \alpha=1.69$} \\
\hline 3.7738 & 0.1449 & 1.3985 & 0.0000 \\
\hline 3.7742 & 0.1653 & 1.1419 & 8.4527 \\
\hline 3.7761 & 0.2098 & 1.1914 & 9.1316 \\
\hline 3.7768 & 0.2433 & 1.2343 & 9.3319 \\
\hline 3.7763 & 0.2778 & 1.2873 & 9.4738 \\
\hline \multicolumn{4}{|c|}{$M=1.20 M_{\odot},[\mathrm{Fe} / \mathrm{H}]=0.25, X=0.70, Y=0.28, Z=0.035, \alpha=1.69$} \\
\hline 3.7640 & 0.0575 & 1.0569 & 0.0000 \\
\hline 3.7686 & 0.1614 & 1.1662 & 9.3975 \\
\hline 3.7689 & 0.2070 & 1.2277 & 9.5663 \\
\hline 3.7664 & 0.2400 & 1.2897 & 9.6682 \\
\hline 3.7662 & 0.2388 & 1.2894 & 9.6693 \\
\hline \multicolumn{4}{|c|}{$M=1.25 M_{\odot},[\mathrm{Fe} / \mathrm{H}]=0.18, X=0.70, Y=0.28, Z=0.030, \alpha=1.69$} \\
\hline 3.7839 & 0.1997 & 1.1361 & 0.0000 \\
\hline 3.7840 & 0.2081 & 1.1466 & 7.5710 \\
\hline 3.7852 & 0.2459 & 1.1910 & 8.9213 \\
\hline 3.7852 & 0.3303 & 1.3126 & 9.4276 \\
\hline \multicolumn{4}{|c|}{$M=1.20 M_{\odot},[\mathrm{Fe} / \mathrm{H}]=0.18, X=0.70, Y=0.28, Z=0.030, \alpha=1.69$} \\
\hline 3.7743 & 0.1125 & 1.0740 & 0.0000 \\
\hline 3.7761 & 0.1603 & 1.1254 & 8.9752 \\
\hline 3.7780 & 0.2095 & 1.1806 & 9.3240 \\
\hline 3.7783 & 0.2454 & 1.2287 & 9.4699 \\
\hline 3.7780 & 0.2620 & 1.2542 & 9.5230 \\
\hline
\end{tabular}


Table 1-Continued

\begin{tabular}{|c|c|c|c|}
\hline $\log T_{\text {eff }}(\mathrm{K})$ & $\log L / L_{\odot}$ & $R / R_{\odot}$ & log Age (yrs.) \\
\hline \multicolumn{4}{|c|}{$M=1.18 M_{\odot},[\mathrm{Fe} / \mathrm{H}]=0.18, X=0.70, Y=0.28, Z=0.030, \alpha=1.69$} \\
\hline 3.7703 & 0.0766 & 0.9611 & 0.0000 \\
\hline 3.7741 & 0.1629 & 0.9444 & 9.2869 \\
\hline 3.7752 & 0.2075 & 0.9396 & 9.4785 \\
\hline 3.7745 & 0.2442 & 0.9427 & 9.5931 \\
\hline 3.7738 & 0.2522 & 0.9457 & 9.6175 \\
\hline \multicolumn{4}{|c|}{$M=1.175 M_{\odot},[\mathrm{Fe} / \mathrm{H}]=0.10, X=0.70, Y=0.28, Z=0.025, \alpha=1.69$} \\
\hline 3.7809 & 0.1295 & 0.9155 & 0.0000 \\
\hline 3.7820 & 0.1661 & 0.9108 & 8.8061 \\
\hline 3.7839 & 0.2104 & 0.9026 & 9.2142 \\
\hline 3.7848 & 0.2450 & 0.8989 & 9.3785 \\
\hline 3.7846 & 0.2766 & 0.8999 & 9.4886 \\
\hline \multicolumn{4}{|c|}{$M=1.15 M_{\odot},[\mathrm{Fe} / \mathrm{H}]=0.10, X=0.70, Y=0.28, Z=0.025, \alpha=1.69$} \\
\hline 3.7758 & 0.0837 & 1.0319 & 0.0000 \\
\hline 3.7793 & 0.1659 & 1.1161 & 9.2446 \\
\hline 3.7807 & 0.2088 & 1.1656 & 9.4358 \\
\hline 3.7806 & 0.2453 & 1.2157 & 9.5502 \\
\hline 3.7780 & 0.2756 & 1.2740 & 9.6401 \\
\hline \multicolumn{4}{|c|}{$M=1.14 M_{\odot},[\mathrm{Fe} / \mathrm{H}]=0.10, X=0.70, Y=0.28, Z=0.025, \alpha=1.69$} \\
\hline 3.7737 & 0.0652 & 1.0199 & 0.0000 \\
\hline 3.7781 & 0.1628 & 1.1183 & 9.3341 \\
\hline 3.7793 & 0.2100 & 1.1743 & 9.5097 \\
\hline 3.7788 & 0.2440 & 1.2240 & 9.6051 \\
\hline 3.7651 & 0.4366 & 1.6273 & 9.8359 \\
\hline \multicolumn{4}{|c|}{$M=1.13 M_{\odot},[\mathrm{Fe} / \mathrm{H}]=0.10, X=0.70, Y=0.28, Z=0.025, \alpha=1.69$} \\
\hline 3.7716 & 0.0465 & 1.0079 & 0.0000 \\
\hline 3.7770 & 0.1663 & 1.1285 & 9.4362 \\
\hline 3.7777 & 0.2100 & 1.1829 & 9.5720 \\
\hline 3.7765 & 0.2439 & 1.2368 & 9.6590 \\
\hline 3.7671 & 0.4040 & 1.5530 & 9.8382 \\
\hline
\end{tabular}


Table 1-Continued

\begin{tabular}{|c|c|c|c|}
\hline $\log T_{\text {eff }}(\mathrm{K})$ & $\log L / L_{\odot}$ & $R / R_{\odot}$ & log Age (yrs.) \\
\hline \multicolumn{4}{|c|}{$M=1.12 M_{\odot},[\mathrm{Fe} / \mathrm{H}]=0.10, X=0.70, Y=0.28, Z=0.025, \alpha=1.69$} \\
\hline 3.7694 & 0.0276 & 0.9649 & 0.0000 \\
\hline 3.7755 & 0.1613 & 0.9381 & 9.4944 \\
\hline 3.7760 & 0.2081 & 0.9363 & 9.6241 \\
\hline 3.7746 & 0.2428 & 0.9422 & 9.7024 \\
\hline 3.7737 & 0.2780 & 0.9462 & 9.7524 \\
\hline \multicolumn{4}{|c|}{$M=1.10 M_{\odot},[\mathrm{Fe} / \mathrm{H}]=0.10, X=0.70, Y=0.28, Z=0.025, \alpha=1.69$} \\
\hline 3.7651 & -0.0105 & 0.9726 & 0.0000 \\
\hline 3.7727 & 0.1619 & 1.1453 & 9.6248 \\
\hline 3.7720 & 0.2071 & 1.2104 & 9.7256 \\
\hline 3.7709 & 0.2453 & 1.2712 & 9.7825 \\
\hline 3.7584 & 0.3918 & 1.5939 & 9.9055 \\
\hline \multicolumn{4}{|c|}{$M=1.15 M_{\odot},[\mathrm{Fe} / \mathrm{H}]=0.00, X=0.70, Y=0.28, Z=0.020, \alpha=1.69$} \\
\hline 3.7891 & 0.1546 & 1.0532 & 0.0000 \\
\hline 3.7892 & 0.1649 & 1.0653 & 7.6434 \\
\hline 3.7908 & 0.2067 & 1.1095 & 8.9677 \\
\hline 3.7923 & 0.2454 & 1.1519 & 9.2369 \\
\hline 3.7926 & 0.3071 & 1.2350 & 9.4692 \\
\hline \multicolumn{4}{|c|}{$M=1.10 M_{\odot},[\mathrm{Fe} / \mathrm{H}]=0.00, X=0.70, Y=0.28, Z=0.020, \alpha=1.69$} \\
\hline 3.7788 & 0.0606 & 0.9911 & 0.0000 \\
\hline 3.7834 & 0.1617 & 1.0899 & 9.3356 \\
\hline 3.7848 & 0.2109 & 1.1460 & 9.5080 \\
\hline 3.7848 & 0.2452 & 1.1923 & 9.5954 \\
\hline 3.7767 & 0.4072 & 1.4910 & 9.8025 \\
\hline \multicolumn{4}{|c|}{$M=1.09 M_{\odot},[\mathrm{Fe} / \mathrm{H}]=0.00, X=0.70, Y=0.28, Z=0.020, \alpha=1.69$} \\
\hline 3.7766 & 0.0413 & 0.9791 & 0.0000 \\
\hline 3.7824 & 0.1653 & 1.0996 & 9.4361 \\
\hline 3.7833 & 0.2054 & 1.1467 & 9.5546 \\
\hline 3.7827 & 0.2459 & 1.2233 & 9.6494 \\
\hline 3.7794 & 0.3397 & 1.3638 & 9.7757 \\
\hline
\end{tabular}


Table 1-Continued

\begin{tabular}{|c|c|c|c|}
\hline $\log T_{\text {eff }}(\mathrm{K})$ & $\log L / L_{\odot}$ & $R / R_{\odot}$ & log Age (yrs.) \\
\hline \multicolumn{4}{|c|}{$M=1.06 M_{\odot},[\mathrm{Fe} / \mathrm{H}]=0.00, X=0.70, Y=0.28, Z=0.020, \alpha=1.69$} \\
\hline 3.7699 & -0.0174 & 0.9438 & 0.0000 \\
\hline 3.7782 & 0.1624 & 1.1173 & 9.6277 \\
\hline 3.7780 & 0.2063 & 1.1763 & 9.7167 \\
\hline 3.7769 & 0.2422 & 1.2322 & 9.7726 \\
\hline 3.7622 & 0.4097 & 1.5989 & 9.9064 \\
\hline \multicolumn{4}{|c|}{$M=1.05 M_{\odot},[\mathrm{Fe} / \mathrm{H}]=0.00, X=0.70, Y=0.28, Z=0.020, \alpha=1.69$} \\
\hline 3.7676 & -0.0372 & 0.9323 & 0.0000 \\
\hline 3.7767 & 0.1661 & 1.1298 & 9.6891 \\
\hline 3.7758 & 0.2085 & 1.1913 & 9.7662 \\
\hline 3.7749 & 0.2434 & 1.2453 & 9.8094 \\
\hline 3.7739 & 0.2736 & 1.2953 & 9.8388 \\
\hline \multicolumn{4}{|c|}{$M=1.04 M_{\odot},[\mathrm{Fe} / \mathrm{H}]=0.00, X=0.70, Y=0.28, Z=0.020, \alpha=1.69$} \\
\hline 3.7653 & -0.0572 & 0.9208 & 0.0000 \\
\hline 3.7749 & 0.1617 & 1.1335 & 9.7308 \\
\hline 3.7739 & 0.2098 & 1.2036 & 9.8072 \\
\hline 3.7731 & 0.2423 & 1.2541 & 9.8419 \\
\hline 3.7716 & 0.2782 & 1.3160 & 9.8732 \\
\hline \multicolumn{4}{|c|}{$M=1.00 M_{\odot},[\mathrm{Fe} / \mathrm{H}]=0.00, X=0.70, Y=0.28, Z=0.020, \alpha=1.69$} \\
\hline 3.7556 & -0.1375 & 0.8778 & 0.0000 \\
\hline 3.7673 & 0.1635 & 1.1760 & 9.8997 \\
\hline 3.7661 & 0.2088 & 1.2460 & 9.9389 \\
\hline 3.7642 & 0.2435 & 1.3085 & 9.9632 \\
\hline 3.7587 & 0.2971 & 1.4274 & 9.9946 \\
\hline \multicolumn{4}{|c|}{$M=1.015 M_{\odot},[\mathrm{Fe} / \mathrm{H}]=-0.09, X=0.70, Y=0.28, Z=0.016, \alpha=1.69$} \\
\hline 3.7725 & 0.9063 & 0.9063 & 0.0000 \\
\hline 3.7819 & 0.1655 & 1.1023 & 9.6838 \\
\hline 3.7816 & 0.2079 & 1.1589 & 9.7557 \\
\hline 3.7806 & 0.2457 & 1.2163 & 9.8035 \\
\hline 3.7713 & 0.3814 & 1.4843 & 9.9038 \\
\hline
\end{tabular}


Table 1-Continued

\begin{tabular}{|c|c|c|c|}
\hline $\log T_{\text {eff }}(\mathrm{K})$ & $\log L / L_{\odot}$ & $R / R_{\odot}$ & log Age (yrs.) \\
\hline \multicolumn{4}{|c|}{$M=0.997 M_{\odot},[\mathrm{Fe} / \mathrm{H}]=-0.09, X=0.70, Y=0.28, Z=0.016, \alpha=1.69$} \\
\hline 3.7682 & -0.0791 & 0.9705 & 0.0000 \\
\hline 3.7790 & 0.1655 & 0.9233 & 9.7695 \\
\hline 3.7782 & 0.2058 & 0.9269 & 9.8252 \\
\hline 3.7773 & 0.2439 & 0.9307 & 9.8619 \\
\hline 3.7683 & 0.3580 & 0.9702 & 9.9372 \\
\hline \multicolumn{4}{|c|}{$M=0.984 M_{\odot},[\mathrm{Fe} / \mathrm{H}]=-0.09, X=0.70, Y=0.28, Z=0.016, \alpha=1.69$} \\
\hline 3.7650 & -0.1058 & 0.8718 & 0.0000 \\
\hline 3.7766 & 0.1611 & 1.1237 & 9.8199 \\
\hline 3.7757 & 0.2081 & 1.1914 & 9.8729 \\
\hline 3.7746 & 0.2437 & 1.2475 & 9.9022 \\
\hline 3.7725 & 0.2819 & 1.3162 & 9.9282 \\
\hline \multicolumn{4}{|c|}{$M=0.948 M_{\odot},[\mathrm{Fe} / \mathrm{H}]=-0.20, X=0.70, Y=0.28, Z=0.0125, \alpha=1.69$} \\
\hline 3.7697 & -0.1160 & 0.9638 & 0.0000 \\
\hline 3.7820 & 0.1652 & 0.9106 & 9.8273 \\
\hline 3.7812 & 0.2101 & 0.9139 & 9.8749 \\
\hline 3.7804 & 0.2429 & 0.9175 & 9.9005 \\
\hline 3.7797 & 0.2594 & 0.9202 & 9.9119 \\
\hline \multicolumn{4}{|c|}{$M=0.921 M_{\odot},[\mathrm{Fe} / \mathrm{H}]=-0.20, X=0.70, Y=0.28, Z=0.0125, \alpha=1.69$} \\
\hline 3.7628 & -0.1736 & 0.8145 & 0.0000 \\
\hline 3.7768 & 0.1660 & 1.1290 & 9.9288 \\
\hline 3.7758 & 0.2085 & 1.1913 & 9.9595 \\
\hline 3.7744 & 0.2389 & 1.2419 & 9.9777 \\
\hline \multicolumn{4}{|c|}{$M=1.10 M_{\odot},[\mathrm{Fe} / \mathrm{H}]=0.00, X=0.72, Y=0.26, Z=0.020, \alpha=1.69$} \\
\hline 3.7696 & 0.0060 & 0.9642 & 0.0000 \\
\hline 3.7771 & 0.1612 & 0.9315 & 9.5681 \\
\hline 3.7777 & 0.2083 & 0.9290 & 9.6760 \\
\hline 3.7765 & 0.2452 & 0.9339 & 9.7467 \\
\hline 3.7726 & 0.3382 & 0.9508 & 9.8459 \\
\hline
\end{tabular}


Table 1-Continued

\begin{tabular}{|c|c|c|c|}
\hline $\log T_{\text {eff }}(\mathrm{K})$ & $\log L / L_{\odot}$ & $R / R_{\odot}$ & log Age (yrs.) \\
\hline \multicolumn{4}{|c|}{$M=1.06 M_{\odot},[\mathrm{Fe} / \mathrm{H}]=0.00, X=0.72, Y=0.26, Z=0.020, \alpha=1.69$} \\
\hline 3.7606 & -0.0707 & 1.0052 & 0.0000 \\
\hline 3.7710 & 0.1623 & 0.9578 & 9.7813 \\
\hline 3.7699 & 0.2104 & 0.9628 & 9.8511 \\
\hline 3.7689 & 0.2462 & 0.9674 & 9.8864 \\
\hline 3.7673 & 0.2795 & 0.9746 & 9.9135 \\
\hline \multicolumn{4}{|c|}{$M=1.06 M_{\odot},[\mathrm{Fe} / \mathrm{H}]=0.00, X=0.68, Y=0.30, Z=0.020, \alpha=1.69$} \\
\hline 3.7793 & 0.0380 & 0.9223 & 0.0000 \\
\hline 3.7849 & 0.1644 & 0.8987 & 9.4339 \\
\hline 3.7856 & 0.2044 & 0.8955 & 9.5502 \\
\hline 3.7845 & 0.2442 & 0.9001 & 9.6443 \\
\hline 3.7800 & 0.3653 & 0.9191 & 9.7847 \\
\hline \multicolumn{4}{|c|}{$M=1.02 M_{\odot},[\mathrm{Fe} / \mathrm{H}]=0.00, X=0.68, Y=0.30, Z=0.020, \alpha=1.69$} \\
\hline 3.7701 & -0.0429 & 0.9620 & 0.0000 \\
\hline 3.7789 & 0.1626 & 0.9237 & 9.6812 \\
\hline 3.7779 & 0.2091 & 0.9281 & 9.7617 \\
\hline 3.7772 & 0.2424 & 0.9311 & 9.7999 \\
\hline 3.7735 & 0.3179 & 0.9470 & 9.8629 \\
\hline \multicolumn{4}{|c|}{$M=1.13 M_{\odot},[\mathrm{Fe} / \mathrm{H}]=0.00, X=0.70, Y=0.28, Z=0.020, \alpha=1.40$} \\
\hline 3.7753 & 0.1165 & 1.0740 & 0.0000 \\
\hline 3.7768 & 0.1621 & 1.1244 & 8.9145 \\
\hline 3.7787 & 0.2094 & 1.1769 & 9.2654 \\
\hline 3.7795 & 0.2454 & 1.2221 & 9.4114 \\
\hline 3.7757 & 0.3133 & 1.3444 & 9.6076 \\
\hline \multicolumn{4}{|c|}{$M=1.06 M_{\odot},[\mathrm{Fe} / \mathrm{H}]=0.00, X=0.70, Y=0.28, Z=0.020, \alpha=1.40$} \\
\hline 3.7600 & -0.0197 & 0.9852 & 0.0000 \\
\hline 3.7671 & 0.1611 & 1.1739 & 9.6296 \\
\hline 3.7662 & 0.2104 & 1.2476 & 9.7287 \\
\hline 3.7648 & 0.2459 & 1.3085 & 9.7803 \\
\hline 3.7595 & 0.3341 & 1.4839 & 9.8609 \\
\hline
\end{tabular}


Table 1-Continued

\begin{tabular}{|c|c|c|c|}
\hline $\log T_{\text {eff }}(\mathrm{K})$ & $\log L / L_{\odot}$ & $R / R_{\odot}$ & log Age (yrs.) \\
\hline \multicolumn{4}{|c|}{$M=1.06 M_{\odot},[\mathrm{Fe} / \mathrm{H}]=0.00, X=0.70, Y=0.28, Z=0.020, \alpha=2.00$} \\
\hline 3.7782 & -0.0148 & 0.9110 & 0.0000 \\
\hline 3.7876 & 0.1649 & 1.0730 & 9.6270 \\
\hline 3.7877 & 0.2081 & 1.1272 & 9.7149 \\
\hline 3.7869 & 0.2438 & 1.1787 & 9.7703 \\
\hline 3.7837 & 0.3374 & 1.3324 & 9.8568 \\
\hline \multicolumn{4}{|c|}{$M=1.00 M_{\odot},[\mathrm{Fe} / \mathrm{H}]=0.00, X=0.70, Y=0.28, Z=0.020, \alpha=2.00$} \\
\hline 3.7648 & -0.1239 & 0.9857 & 0.0000 \\
\hline 3.7786 & 0.1636 & 0.9252 & 9.8762 \\
\hline 3.7780 & 0.2107 & 0.9278 & 9.9198 \\
\hline 3.7768 & 0.2444 & 0.9326 & 9.9445 \\
\hline 3.7760 & 0.2596 & 0.9361 & 9.9545 \\
\hline \multicolumn{4}{|c|}{$M=1.089 M_{\odot},[\mathrm{Fe} / \mathrm{H}]=0.00, X=0.70, Y=0.28, Z=0.020, \alpha=1.55$} \\
\hline 3.7719 & 0.0385 & 0.9540 & 0.0000 \\
\hline 3.7773 & 0.1631 & 0.9304 & 9.4391 \\
\hline 3.7782 & 0.2093 & 0.9267 & 9.5725 \\
\hline 3.7775 & 0.2440 & 0.9299 & 9.6523 \\
\hline 3.7714 & 0.3690 & 0.9563 & 9.8047 \\
\hline \multicolumn{4}{|c|}{$M=1.06 M_{\odot},[\mathrm{Fe} / \mathrm{H}]=0.00, X=0.70, Y=0.28, Z=0.020, \alpha=1.55$} \\
\hline 3.7654 & -0.0186 & 0.9828 & 0.0000 \\
\hline 3.7731 & 0.1617 & 0.9486 & 9.6286 \\
\hline 3.7728 & 0.2059 & 0.9502 & 9.7180 \\
\hline 3.7714 & 0.2466 & 0.9564 & 9.7793 \\
\hline 3.7667 & 0.3351 & 0.9770 & 9.8599 \\
\hline \multicolumn{4}{|c|}{$M=1.06 M_{\odot},[\mathrm{Fe} / \mathrm{H}]=0.00, X=0.70, Y=0.28, Z=0.020, \alpha=1.85$} \\
\hline 3.7745 & -0.0160 & 0.9255 & 0.0000 \\
\hline 3.7833 & 0.1639 & 1.0934 & 9.6279 \\
\hline 3.7833 & 0.2073 & 1.1494 & 9.7159 \\
\hline 3.7822 & 0.2430 & 1.2034 & 9.7714 \\
\hline 3.7787 & 0.3364 & 1.3618 & 9.8578 \\
\hline
\end{tabular}


Table 1 - Continued

\begin{tabular}{rlll}
\hline \hline $\log T_{\text {eff }}(\mathrm{K})$ & $\log L / L_{\odot}$ & $R / R_{\odot}$ & $\log$ Age (yrs.) \\
\hline$M=1.033 M_{\odot},[\mathrm{Fe} / \mathrm{H}]=0.00$, & $X=0.70$, & $Y=0.28$, & $Z=0.020, \alpha=1.85$ \\
\hline 3.7681 & -0.0696 & 0.8962 & 0.0000 \\
3.7789 & 0.1626 & 1.1138 & 9.7623 \\
3.7782 & 0.2090 & 1.1788 & 9.8291 \\
3.7774 & 0.2453 & 1.2334 & 9.8647 \\
3.7748 & 0.3000 & 1.3297 & 9.9067 \\
\hline
\end{tabular}

Note. - For each track of particular mass, metallicity, and mixinglength parameter, five points on the evolutionary track are listed. The first point denotes the zero-age main sequence, and the last marks the end of the modeling sequence, while the three others correspond to the minimum, central, and maximum luminosities of our error box $\left(\log L / L_{\odot}=0.164\right.$, $\log L / L_{\odot}=0.204, \log L / L_{\odot}=0.244$, respectively). In some cases, fewer than five points are given for tracks starting out with high luminosity. 


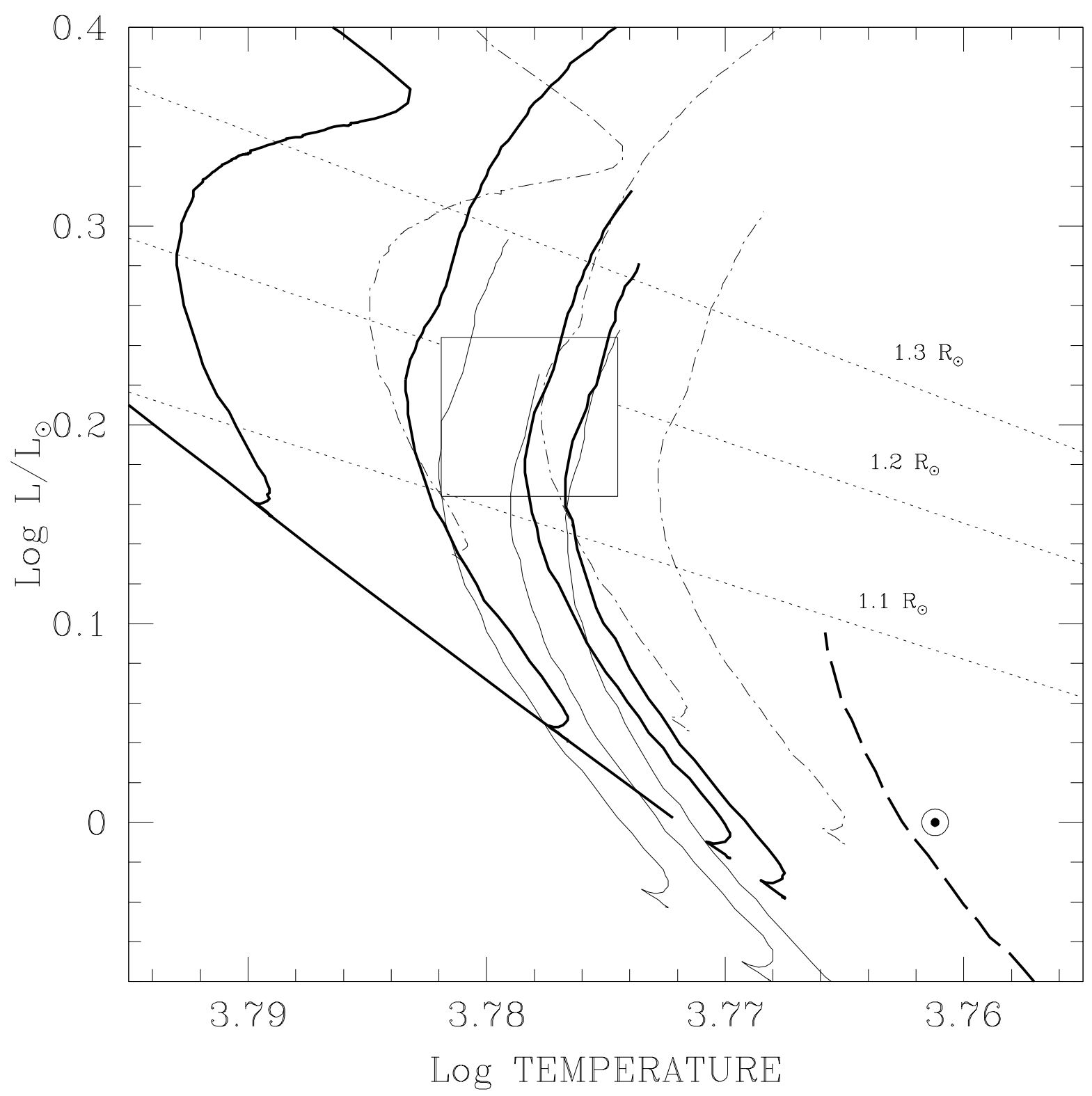

Fig. 1.- A small section of the Hertzsprung-Russell diagram in the vicinity of HD 209458 and the Sun. Our computed evolution tracks are shown for $Z=0.02$ (thick solid lines), for $Z=0.016$ (thin solid lines), and for $Z=0.025$ (dot-dash lines). Masses range from 1.05 to $1.15 M_{\odot}$ for $Z=0.02$ (see text). The long-dashed track is the theoretical evolution of the Sun, while the dot near it marks the known solar temperature and luminosity values. All tracks that do not run off the plot are stopped at 7 Gyr. Three lines of constant radius are shown as well. 


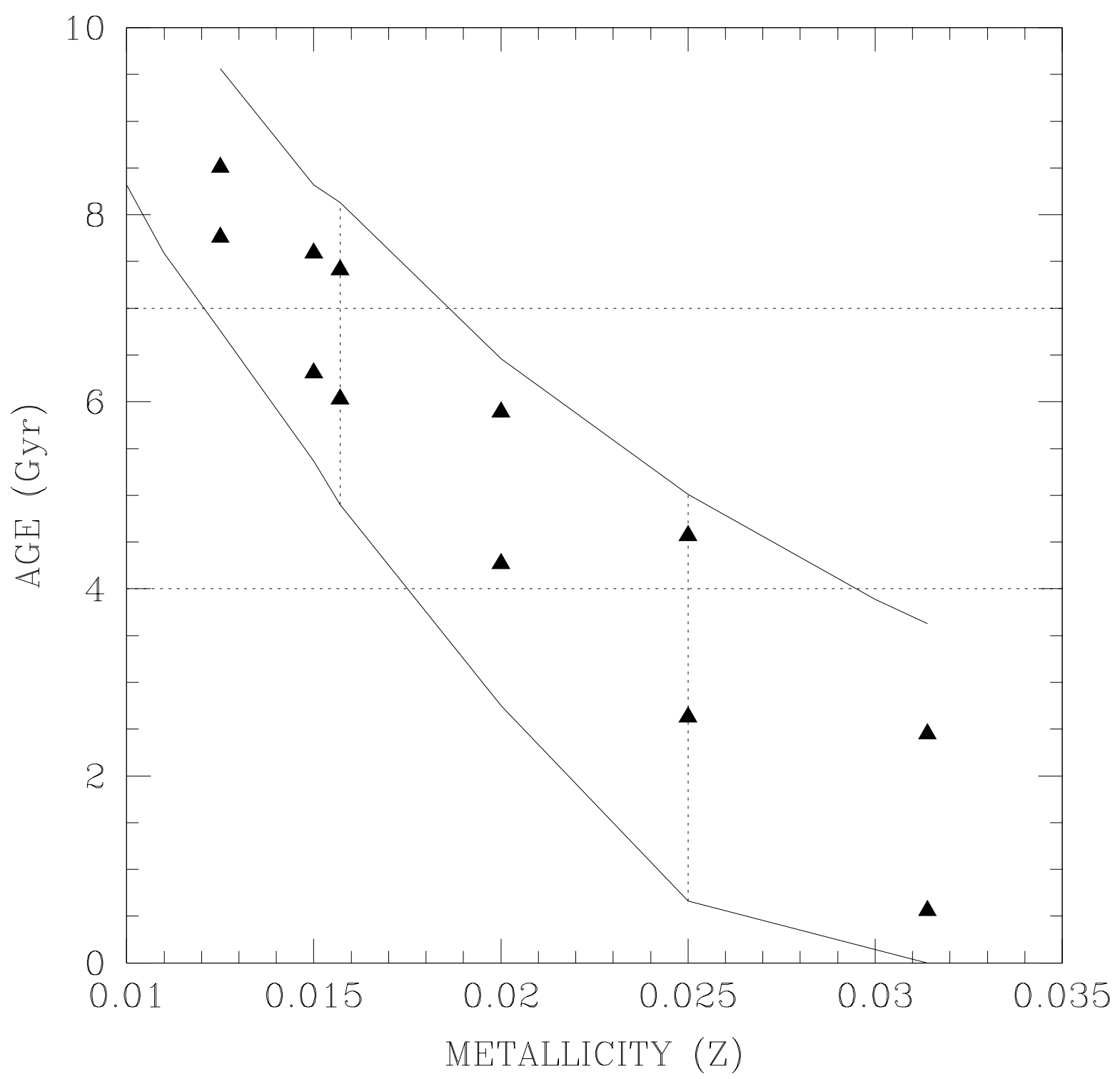

Fig. 2.- The correlation between stellar metallicity and computed age. For selected metallicities, two triangles denote the ages at which the "best" stellar model (the one that achieves the target values $T_{\text {eff }}=6000 \mathrm{~K}$ and $L / L_{\odot}=1.61$ ) evolves through the limits of the temperature/luminosity error range for HD 209458. The curves illustrate maximum and minimum possible ages over all models, and horizontal lines mark our preferred age bounds used in Figs. $3 \& 4$. 


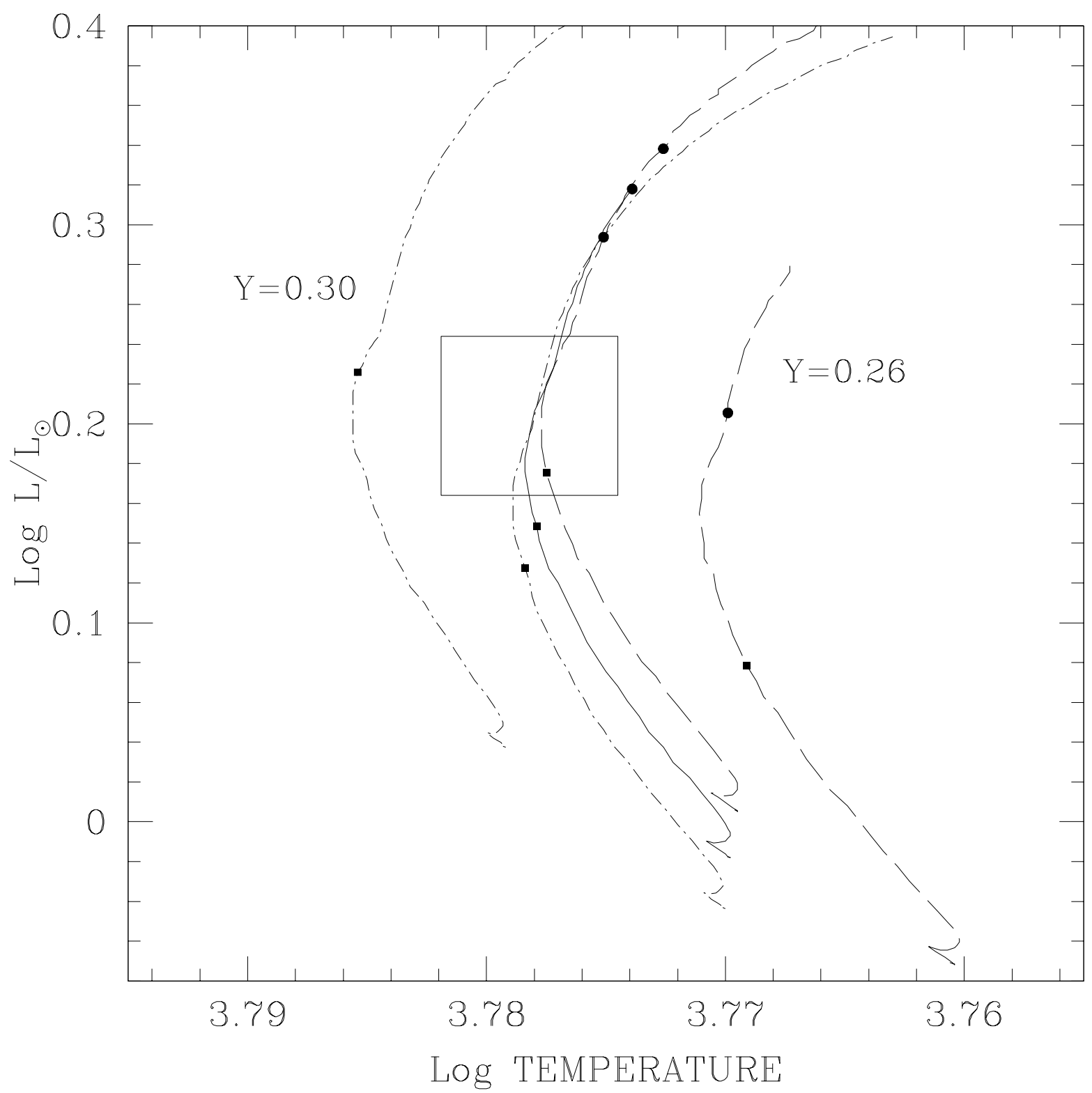

Fig. 3.- The effect of changing the initial helium abundance on the models in the same region of the H-R diagram shown in Fig. 1. The patterns trace tracks of fixed $Y$-value (as marked), and the central, unbroken curve corresponds to the most favored model of $Y=0.28$, $M=1.06 M_{\odot}$. The middle $Y=0.26$ model has mass $1.10 M_{\odot}$, while the middle $Y=0.30$ model has mass $1.02 M_{\odot}$; the two on the outside are both $1.06 M_{\odot}$. All models are for a metallicity $Z=0.02$. In addition, ages are delimited by squares and circles, which indicate the 4 -Gyr and the 7-Gyr points, respectively. 


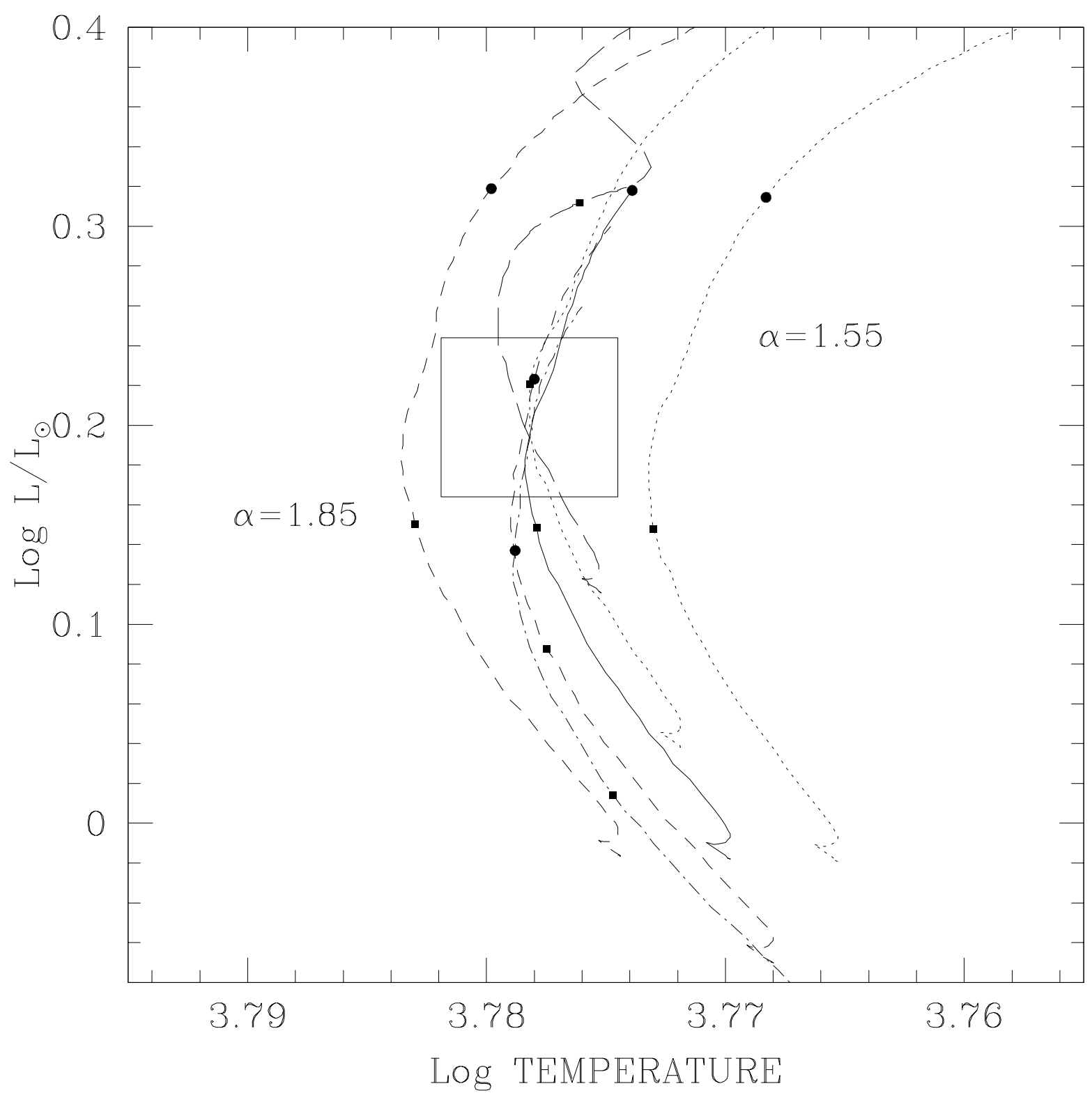

Fig. 4.- The effect of changing the mixing length parameter on the models in the same region of the H-R diagram shown in Fig. 1. All models plotted here have abundances $Z=0.02$ and $Y=0.28$. The dotted and short-dashed curves have $\alpha$-values as marked, the dot-dash pattern traces a model of $\alpha=2.00, M=1.01 M_{\odot}$, and the long-dashed track indicates a model with $\alpha=1.40, M=1.13 M_{\odot}$. The solid curve is a track maintaining the solar $\alpha$ (1.69) and $1.06 M_{\odot}$, while the inner $\alpha=1.55$ and $\alpha=1.85$ tracks have had their masses shifted to $1.09 M_{\odot}$ and $1.03 M_{\odot}$, respectively. The two outside tracks shown are $1.06 M_{\odot}$. As in figure 3, squares mark the 4 Gyr point, and circles denote 7 Gyr. 


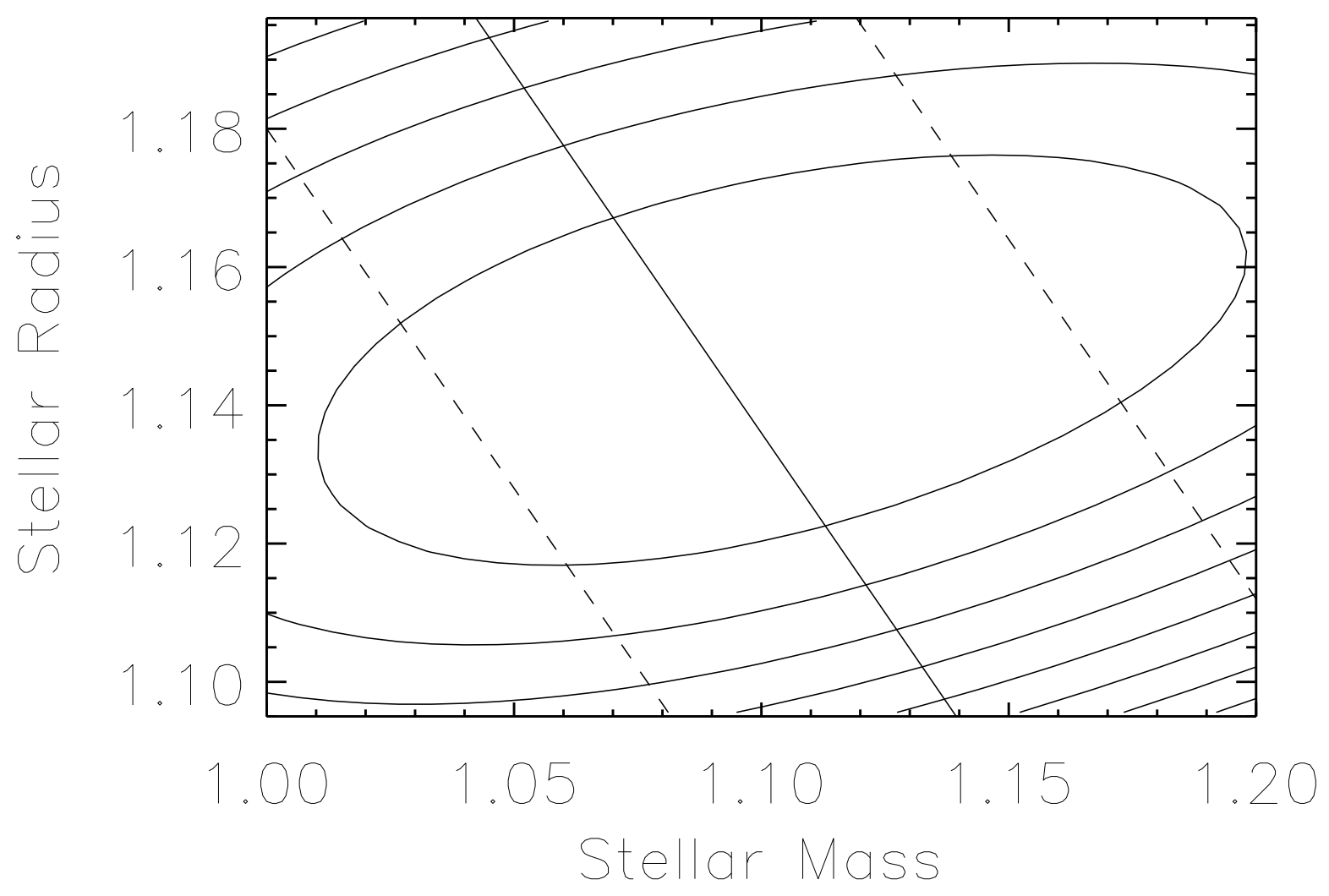

Fig. 5.- The astrophysical relation between stellar mass and radius (solid straight line) breaks the degeneracy in the transit light curve solution, which produces an almost orthogonal relation (curved contours) taken from Brown et al. (2001). All units are solar. 